\title{
Fabrication of a capacitive relative humidity sensor using aluminum thin films deposited on etched printed circuit board
}

\author{
Jacqueline Ann L. Lee, and Ivan B. Culaba \\ Department of Physics, Ateneo de Manila University, Loyola Heights, Quezon City, Philippines, 1108
}

\begin{abstract}
A capacitive humidity-sensing device was created by thermal evaporation of $99.999 \%$ aluminum. The substrate used for the coating was etched double-sided printed circuit board. The etched printed circuit board serves as the dielectric of the capacitor while the aluminum thin films deposited on either side serve as the plates of the capacitor. The capacitance was measured before and after exposure to humidity. The device was then calibrated by comparing the readings of capacitance with that of the relative humidity sensor of the Vernier LabQuest2. It was found that there is a linear relationship between the capacitance and relative humidity given by the equation $\mathrm{C}=1.418 \mathrm{RH}+29.139$ where $\mathrm{C}$ is the capacitance and $\mathrm{RH}$ is the relative humidity. The surface of the aluminum films is porous and it is through these pores that water is adsorbed and capillary condensation occurs, thereby causing the capacitance to change upon to exposure humidity.
\end{abstract}

\section{Introduction}

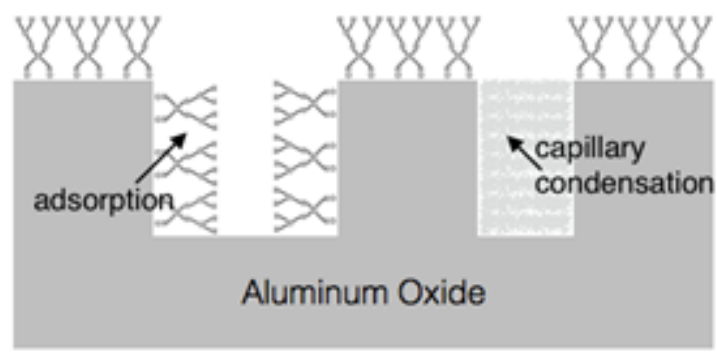

Figure 1. Adsorption and capillary condensation on the film.

Humidity sensors are used for numerous applications in various fields. Capacitive humidity sensors use changes in the capacitance reading to indicate the change in the moisture content of the air. In these sensors, dielectric thin films are deposited on a substrate. When there is a change in the humidity, the moisture in the air is taken in by the thin film. This changes the dielectric constant and indicates, in the change of capacitance, the change in the humidity. Metal oxide thin films are also used in creating these capacitive humidity sensors. The metal oxide thin films are made to be porous using various techniques such as glancing angle deposition wherein the substrate is rotated while the film is being deposited [1]. These films sense the changes in humidity through its pores, which are filled with water molecules, and thus its dielectric constant and capacitance changes. This is due to capillary condensation in the pores and adsorption of water on the surface of the film [2], which causes the electrical properties of the device to change as shown in the change in capacitance. A schematic for capillary condensation and adsorption is shown in Figure 1.

The other methods for fabricating these sensors are quite complex and involve complicated processes to create the porous surface needed. The novelty of the method presented in this paper is that it makes use of a simpler method of creating the porous structures. It is a one step process where the film has to simply be deposited at a normal incidence to the substrate. No intermediate steps are needed to ensure the formation of a porous film.

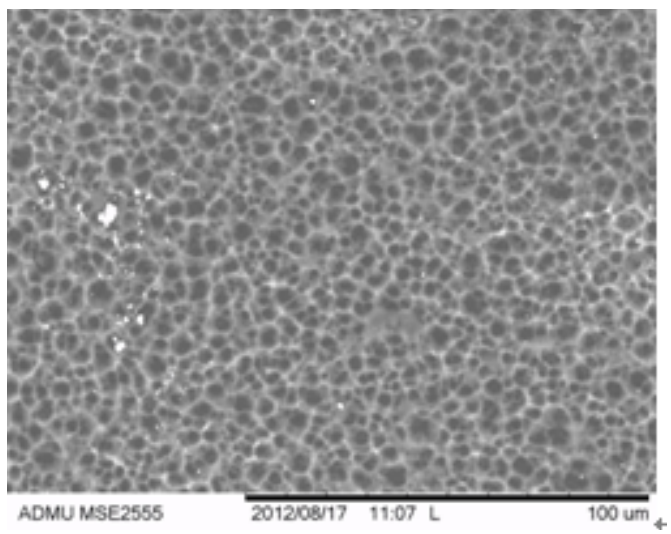

Figure 2. (a) SEM image of etched PCB. From: E.D. Dumayaca, N.N. Garcia, I.B. Culaba. Proceedings $30^{\text {th }}$ Physics Congress of the Samahang Pisikang Pilipinas. 22 (2012) 


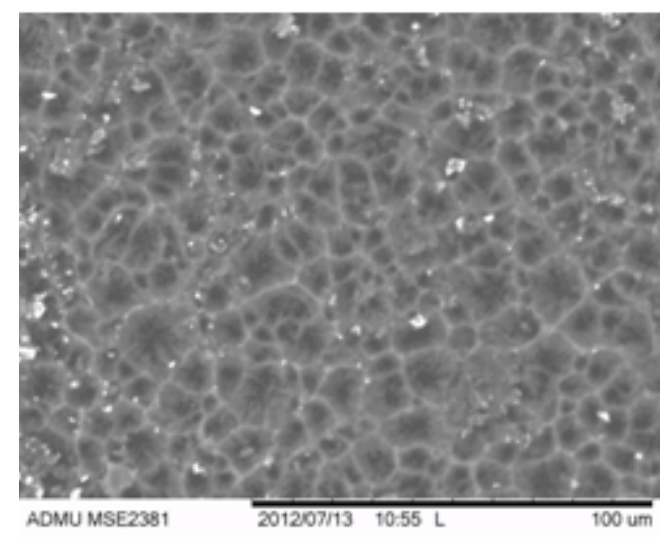

Figure 2. (b) SEM image of aluminum coated etched PCB. From: E.D. Dumayaca, N.N. Garcia, I.B. Culaba. Proceedings $30^{\text {th }}$ Physics Congress of the Samahang Pisika ng Pilipinas. 22 (2012)

From a study on the morphology of aluminum thin films deposited on etched printed circuit board, it was found that the surface of the etched printed circuit board was porous. After coating the surface with aluminum, it was found that the porous surface of the printed circuit board was retained [3] as shown in figures 2 (a) and 2 (b). The use of the already porous surface of etched printed circuit board, as the substrate for the coatings, produces a porous film and allows an easier method for constructing the capacitive humidity sensor.

In this paper, aluminum thin films deposited on etched printed circuit board is used as a capacitor. The aluminum thin films serve as the plates of the capacitor while the etched printed circuit board serves as the dielectric between the plates. This capacitor was used as a relative humidity sensor and its response to humidity was studied and compared to a commercially available sensor.

\section{Methodology}

Preparation of Substrates. To create the capacitor, the printed circuit board (PCB) is prepared. This will serve as the substrate on which the aluminum thin film will be deposited. First, double sided printed circuit board is cut into $1.27 \mathrm{~cm} \mathrm{x} 10.16 \mathrm{~cm}(0.5$ " $\mathrm{c} 4.0$ ") pieces and holes were drilled onto each end. One of the holes is drilled $1.75 \mathrm{~cm}$ from one end and the other is drilled $0.50 \mathrm{~cm}$ from the opposite end as shown in figure 3. This will allow bolts to be attached and it will be used to measure the capacitance.

Then the cut pieces of printed circuit board were submerged in ferric chloride $\left(\mathrm{FeCl}_{3}\right)$ to remove the copper on the surface. Once the copper was removed, the etched printed circuit board was rinsed with distilled water and was subjected to ultrasonic cleaning using the Fisher Scientific Ultrasonic Cleaner FS30H. They were further cleaned using isopropyl alcohol and Kimwipes ${ }^{\circledR}$. Lastly, the etched PCB was masked with tape as shown in figure 3. The unmasked areas will be coated with aluminum while the masked areas will remain uncoated.

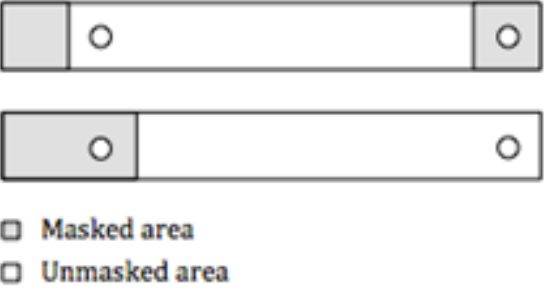

Figure 3. Masked areas and position of holes on the etched PCB

Coating Substrates with Aluminum. The Kinney vacuum system was used for coating the etched PCB. The substrates were placed on a rotating substrate holder inside the vacuum chamber. To coat the etched printed circuit board, 2 tungsten coils were attached to the current sources inside the vacuum chamber. On each coil, 20 pieces on $99.999 \%$ aluminum wires were attached. The coating was done when the pressure of the chamber reached $2.5 \times 10^{-5} \mathrm{mmHg}$. To start the coating process, the first coil was heated up by applying a current. The current was gradually increased until it reached 60 amperes and the first side of the etched printed circuit board was exposed to the aluminum vapor for 3 minutes. The thickness of the film was then measured using a thickness monitor. After this, the substrate holder is rotated to prepare the second side for coating. The same procedure is applied to the second coil so that the second side is coated.

Assembling the sensor. Each aluminium coated PCB was fitted with nuts, bolts and washers to serve as contact points for the capacitor as shown in figure 4(a).

Four of these capacitors are connected in parallel to form the humidity sensor as shown in figure 4(b). The capacitance is then measured using an AC-2000 Digital Multi-meter.

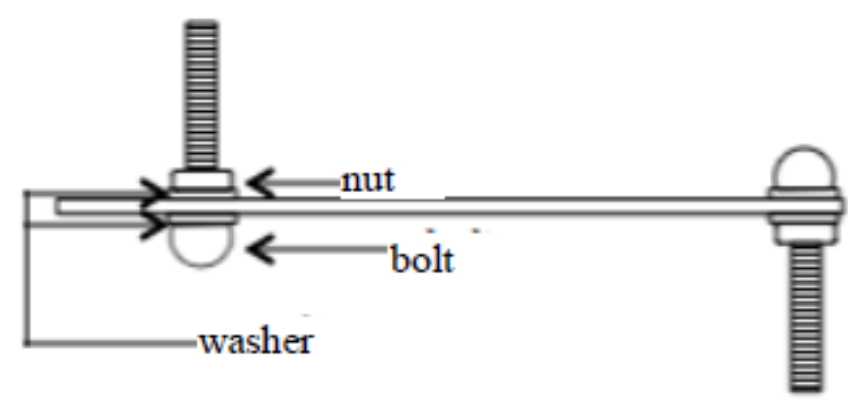

Figure 4. (a) Capacitor assembly 


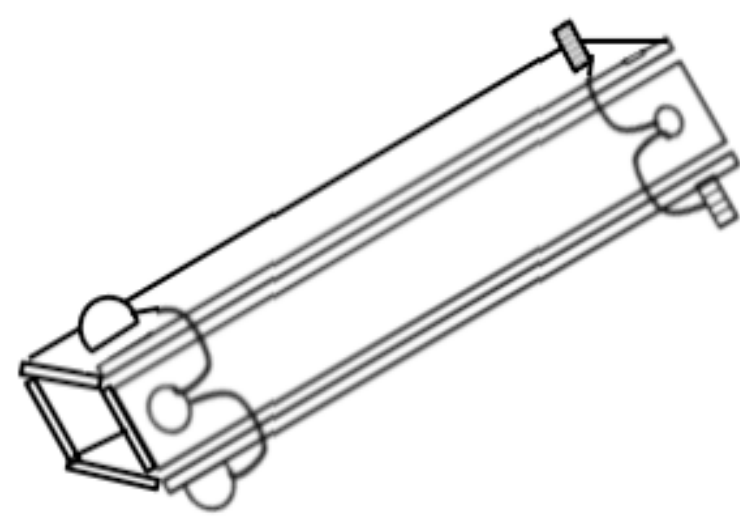

Figure 4. (b) Relative humidity sensor

Calibrating the Sensor. The sensor is calibrated by simultaneously measuring the capacitance of the sensor, using an AC-2000 Digital Multi-meter, and the relative humidity, using the relative humidity sensor of the Vernier LabQuest2. Both sensors are placed inside the same container and the humidity inside the container is decreased as capacitance and relative humidity are collected.

The capacitance is then plotted against relative humidity and the equation of the curve is obtained.

\section{Results and Discussion}

The thickness of the films for each side of the capacitor was measured to be $440 \AA$ and $480 \AA$.

The capacitance readings obtained over time as the humidity is changed is shown in figure 5 while the change of relative humidity over time is shown in figure 6. It can be seen from figures 5 and 6 that there is a similar decreasing trend for both the capacitance versus time graph and the relative humidity versus time graph.

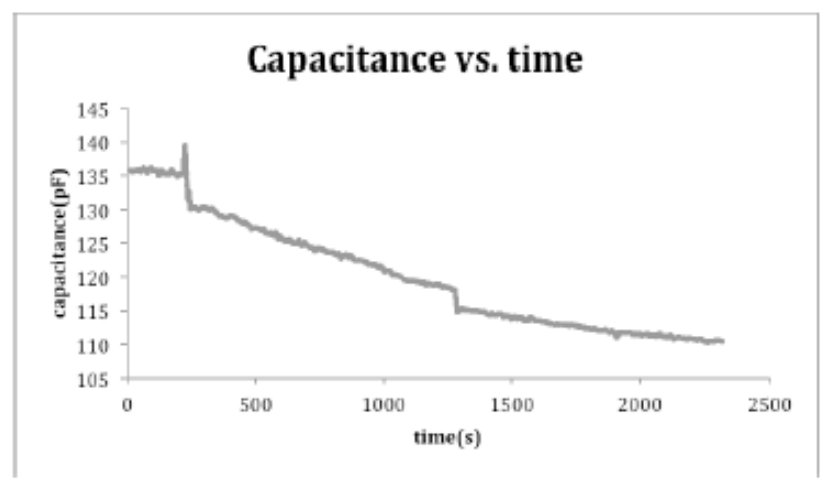

Figure 5. Graph of capacitance over time.

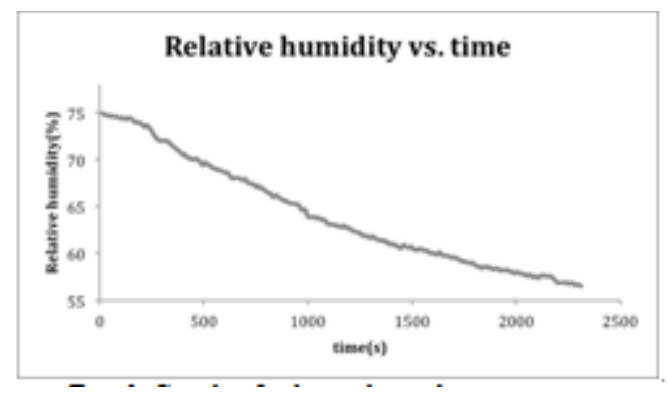

Figure 6. Graph of relative humidity over time.

Comparing the capacitance and relative humidity in figure 7 shows that there is a linear relationship between the relative humidity and the capacitance of the device. By obtaining the best-fit line (eq. 1) of the data points where $\mathrm{C}$ is the capacitance and $\mathrm{RH}$ is the relative humidity, the capacitance measured from the device can be easily converted to the relative humidity value and vice versa.

$C=$

$1.418 R H$

29.139 .

(1)

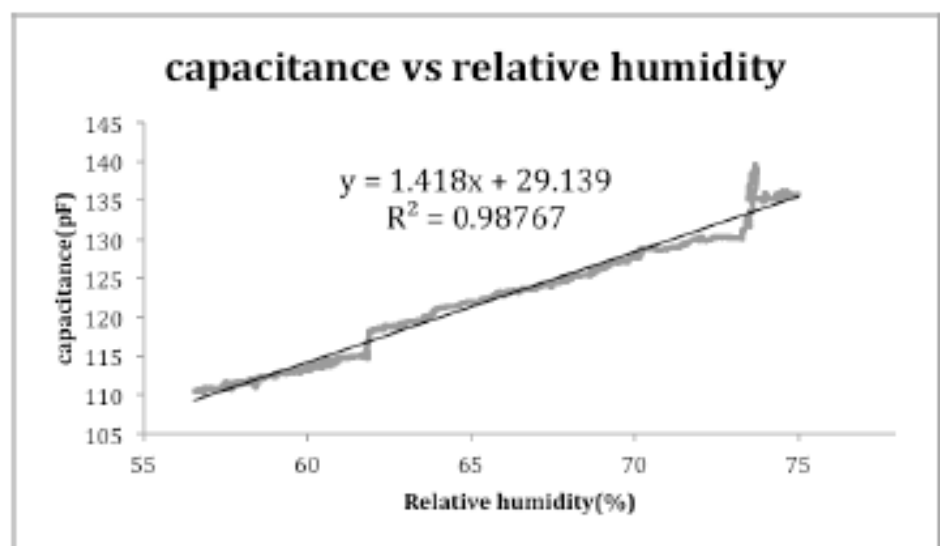

Figure 7. Graph of capacitance vs. relative humidity

When the alumimum coating is exposed to the air, an oxide layer is formed. The oxide layer enables the water molecules in the air to be adsorbed on the surface [4] as shown figure 8 . The pores of the film are also filled by the water molecules through capillary condensation as shown previously in figure 1 . This changes the electrical properties of the aluminum films and thus, changes the measured capacitance depending on the amount of adsorbed water. Water can also be taken in by the porous etched PCB and cause a change in the dielectric constant, and thus, the measured capacitance. As the relative humidity is increased, the amount of water molecules that that are adsorbed on the film surface increases and the amount of water molecules that enter the pores of the dielectric increases, thereby increasing the capacitance reading.

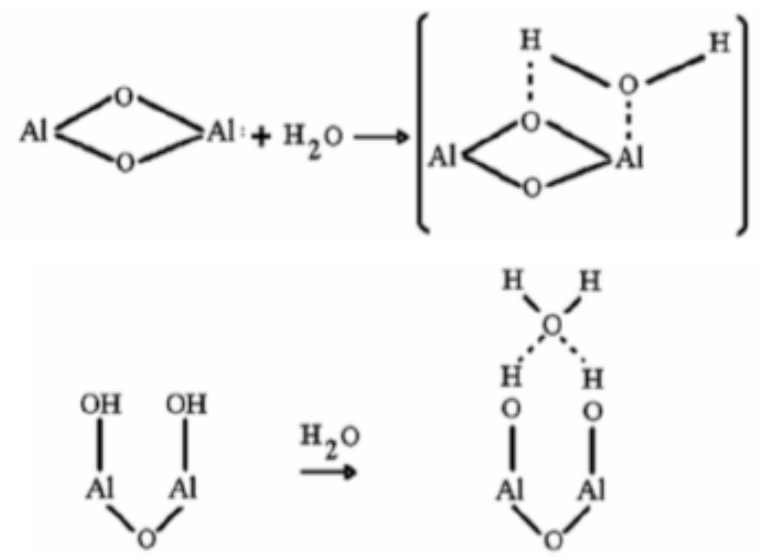

Figure 8. Adsorption of water on aluminum oxide. 


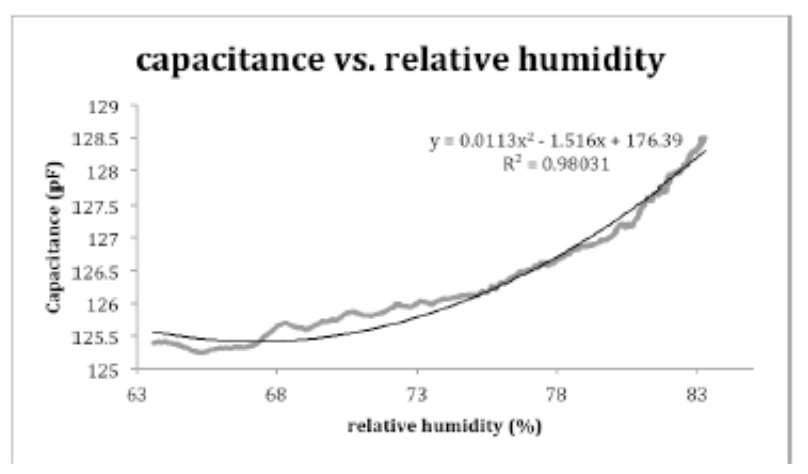

Figure 9. Graph of capacitance vs. relative humidity for the aged capacitor

After aging the sensor for three months, it was found that it no longer retained its original sensing properties. The capacitance no longer varied linearly with relative humidity as shown in figure 9. There is very little increase in capacitance as the relative humidity was increased. There was, approximately, only a $3 \mathrm{pF}$ change in the capacitance as the relative humidity was increased from $63 \%$ to $83 \%$ for the aged sensor compared to the, approximately, $25 \mathrm{pF}$ change in capacitance as the humidity was changed from $56 \%$ to $75 \%$ for the nonaged sensor. This could be attributed to contaminants in the pores that prevent the adsorption of water and capillary condensation; thus, hindering the sensor from responding to the humidity changes in the environment.

\section{Conclusion}

A capacitive relative humidity sensor was fabricated by depositing aluminum thin films on either side of etched double-sided printed circuit board. Exposing the sensor to humidity causes water molecules to fill the pores of the film and the dielectric. Water molecules are also adsorbed in the aluminum oxide surface, which causes a $\mathrm{r}$ change in the capacitance reading. By comparing the readings of capacitance of the non-aged device with that of the relative humidity sensor of the Vernier LabQuest2, the device was calibrated. It was found that there is a linear relationship between the capacitance and relative humidity given by the equation $\mathrm{C}=1.418 \mathrm{RH}+29.139$ where $\mathrm{C}$ is the capacitance and $\mathrm{RH}$ is the relative humidity.

\section{References}

1. J. J. Steele, G. A. Fitzpatrick, M. J. Brett, IEEE Sensors J. 6 (2007) 24-27

2. O. K. Varghese, C. A. Grimes, J. Nanosci. Nanotech. 3 (2003) 277-293

3. E.D. Dumayaca, N.N. Garcia, I.B. Culaba, Proceedings 30th Physics Congress of the Samahang Pisika ng Pilipinas. (2012.)

4. T. Morimoto, M. Nagao, F. Tokuda, J. Phys. Chem. 73 (1969) 243-248

5. V. Timár-Horváth, L. Juhász, A. Vass-Várnai, G. Perlaky, Microsyst. Technol. 14 (2008) 1081-1086

6. F. Casanova, C. E. Chiang, C. P. Li, I. V. Roshchin, A. M. Ruminski, M. J. Sailor, I. K. Schuller, Nanotechnology. 19 (2008)

7. T. Morimoto, M. Nagao, F. Tokuda, Bull. Chem. Soc. Jpn. 44 (1971) 1282-1288

8. J. J. Steele, G. A. Fitzpatrick, M. J. Brett, IEEE Sensors J. 7 (2007) 955-956

9. J. Li, Y. Liu, M. Tang, J. Li, X. Lin, IET Micro Nano Lett. 7 (2012) 1097-1100

10. H. Farahani, R. Wagiran, M. N. Hamidon, Sensors, 14 (2014) 7881-7939 